\title{
DIFFUSION MECHANISM OF PARTICLE BEAMS IN THE PRESENCE OF PHASE MODULATION IN DOUBLE RF SYSTEMS*
}

\author{
M. Ball, J. Budnick, C. M. Chu ${ }^{\dagger}$, K. M. Fung, B. Hamilton, D. Jeon, X. Kang, L. L. Kiang, \\ S. Y. Lee, A. Pei, T. Sloan, Indiana University, Bloomington, IN \\ K. Y. Ng, FNAL, Batavia, IL
}

\section{Abstract}

The particle diffusion mechanism due to phase modulation on one of the rf cavities in a double rf system is studied. We find that the Einstein relation is satisfied if there is a global chaos in the phase space. On the other hand, the existence of dominant parametric resonances forces the particles streaming along the separatrix, which may result in anomalous diffusion process. The particle tracking simulations based on the Hamiltonian for the double rf system is employed to reveal the essential diffusion mechanism. Also for the first time, a coherent octuple mode has been observed in the bunch beam excitation. The phase space evolution of the octuple mode is displayed.

\section{INTRODUCTION}

Controlled particle beam diffusion has many applications in beam physics such as phase space painting for beam injection, beam dilution for minimizing the growth due to the collective instabilities, stochastic beam scraping and stochastic slow beam extraction. Practically, the diffusion process is dominated by the chaos generated by overlapping resonances[1]. Particles in the existence of Langevin force diffuse in the phase space following the Einstein relation: $\sigma^{2} \simeq \mathcal{D} t$, where $\sigma^{2}$ is the mean square of the beam bunch, $\mathcal{D}$ is the diffusion coefficient and $t$ is the evolving time. However, there are anomalous diffusion processes that do not follow the Einstein relation. They occur in plasma physics, Lévy dynamics, turbulent flow and space charge dominated beams [2]. Understanding the causes and detail dynamics of the anomalous diffusion is particularly important in those areas.

Experimentally, We studied the particle diffusion processes in phase space by applying a phase modulation on one on a double rf system at the Indiana University $\mathrm{Cy}-$ clotron Facility (IUCF) Cooler ring. The experiment data alone with in-depth beam tracking simulation will be presented in this paper.

\footnotetext{
* Work supported by U.S. Department of Energy DE-FG0292ER40747 and National Science Foundation PHY-9512832.

† Email: cmchu@iucf.indiana.edu
}

\section{SYNCHROTRON EQUATION OF MOTION}

The normalized conjugate phase space coordinates are defined as

$$
\begin{aligned}
\phi & =-h_{1}\left(\theta-\theta_{\mathrm{s}}\right) \\
\delta & =\left(h_{1}|\eta| / \nu_{\mathrm{s}}\right)\left(\Delta p / p_{0}\right)
\end{aligned}
$$

where $h_{1}$ is the harmonic number of the rf cavity, $\theta$ and $\theta_{\mathrm{s}}$ are respectively the orbiting angles of a non-synchronous and synchronous particles, $\eta$ is the phase slip factor, $p_{0}$ is the momentum of a synchronous particle, $\Delta p=p-p_{0}$ is the momentum deviation, $\nu_{\mathrm{s}}=\left(h_{1} e V|\eta| / 2 \pi \beta^{2} E\right)^{1 / 2}$ is the synchrotron tune, $V$ is the voltage, $e$ is the particle's electric charge, and $\beta$ and $E$ are the Lorentz velocity factor and energy of the particle. the synchrotron motion of a particle in a double-rf system with phase modulation can then be written as

$$
\begin{aligned}
\dot{\delta}= & -\nu_{s}\left[\sin \left(\phi+\Delta \phi_{1}\right)-r \sin \left(h \phi+\Delta \phi_{2}\right)\right] \\
& -\lambda \delta+D \xi \\
\dot{\phi}= & \nu_{s} \delta
\end{aligned}
$$

where $r=V_{2} / V_{1}$ is the ratio of the primary and secondary rf voltages, $h=h_{2} / h_{1}$ is the ratio of harmonic numbers where $V_{1}$ and $V_{2}$ are the voltage gains for the primary and secondary rf systems, respectively, $h_{1}$ and $h_{2}$ are the harmonic numbers for the primary and secondary rf system, respectively, $\lambda$ is the damping decrement, the white Gaussian noise $\xi$ where $\left\langle\xi(\theta) \xi\left(\theta^{\prime}\right)\right\rangle=\delta\left(\theta-\theta^{\prime}\right)$ with $\langle\xi\rangle=0$, and $D$ is the amplitude of the white noise. At the Cooler ring, typically $\lambda$ and $D$ are set about $3 \times 10^{-6}$ and $2 \times 10^{-4}$. The phase modulation term in Eq. 3 is given by

$$
\begin{aligned}
\Delta \phi_{1}(t) & =A_{1} \sin \left(\nu_{\mathrm{m} 1} \theta\right), \\
\Delta \phi_{2}(t) & =A_{2} \sin \left(\nu_{\mathrm{m} 2} \theta\right)+\Delta \phi_{0}
\end{aligned}
$$

where $A_{i}$ and $\nu_{\mathrm{m} i}=f_{\mathrm{m} i} / f_{s}$ are the modulation amplitudes and the modulation tunes, respectively, where $i=1$ and $i=2$ represent primary and secondary cavities, respectively, $f_{\mathrm{m} i}$ is the modulation frequency and $f_{s}$ is the synchrotron frequency with primary cavity alone; and $\Delta \phi_{0}$ is a relative phase difference between the two rf systems. Without damping and fluctuation terms, the double rf system with phase modulation can be described in the following Hamiltonian form

$$
\begin{aligned}
\tilde{H}_{0}= & \frac{1}{2} \nu_{s} \delta^{2}+\nu_{s}\left\{1-\cos \left(\phi+\Delta \phi_{1}\right)\right. \\
& \left.+\frac{r}{h}\left[1-\cos \left(h \phi+\Delta \phi_{2}\right)\right]\right\} .
\end{aligned}
$$


Multi-particle tracking simulation follows Eq.3 and 4. We focus on several important factors, namely, mean square of beam width, averaged beam center and the Poincaré surfaces of section of the system.

\section{EXPERIMENTS}

The main purpose for our experiment was to systematically study the beam dilution mechanism in the presence of phase modulation of a double rf system. The IUCF Cooler ring is a 6-sided proton storage ring with electron cooling and two rf acceleration cavities. The primary MPI cavity operates at a harmonic number $h_{1}=1$, and the secondary PPA cavity operates at the harmonic number $h_{2}=9$. The ring can accelerate protons from 45 to $500 \mathrm{MeV}$ in kinetic energy. In this experiment, the beam current was between 100 to $500 \mu \mathrm{A}$ for a $45 \mathrm{MeV}$ single bunch proton beam in the ring.

After injection, the proton beam was cooled by an electron beam cooling system to reduce its momentum spread and transverse emittances. The electron cooling rate for the Cooler ring was measured to be about $3 \pm 1 \mathrm{~s}^{-1}$ at this energy [3]. The accelerator cycle time was set at $10 \mathrm{~s}$, and the secondary rf system and the data acquisition system were turned on $3 \mathrm{~s}$ after the completion of injection, which was much longer than the needed $300 \mathrm{~ms}$ cooling time. The cooled beam bunch rms length was about $15 \sim 20$ ns with a momentum spread less than $0.1 \%$. The primary rf voltage was set at about $300 \mathrm{~V}$, which resulted in a synchrotron frequency, $f_{\mathrm{s}}$, of about $705 \mathrm{~Hz}$ while operating with the primary rf cavity alone.

Beam profile was taken from a BPM sum signal passing through a low loss cable, and recorded by a fast digital scope which was set at a sampling rate of $1 \mathrm{GHz}$ for a total of 512 or 1024 channels for each turn. The bunch profiles were digitized every 25 to 75 turns. A pretrigger to start data recording was set at least $100 \mathrm{~ns}$ prior to the arrival of the beam bunch. This sampling rate can provide the detailed evolution of beam profile in the diffusion process.

\section{DATA ANALYSIS}

We tried to understand the diffusion mechanism by looking at physics quantities such as mean square of longitudinal beam size, Fast Fourier Transform (FFT) spectra of mean square beam size and averaged beam center position and actual beam evolution profiles. The evolution of the beam profile can be characterized by the mean square bunch length $<\sigma^{2}>$ which is defined as

$$
<\sigma^{2}>=\frac{1}{N} \sum_{j=1}^{N}\left(\phi_{j}-\phi_{\mathrm{avg}}\right)^{2},
$$

where $N$ is the number of particles in a beam bunch and $\phi_{\text {avg }}=\frac{1}{N} \sum_{j=1}^{N} \phi_{j}$ is the average value of $\phi$ and where $\phi_{j}$ is the $\phi$ value of the $j$-th particle.

Fig. 1 summarizes our data for modulation on the secondary rf cavity, where the final beam width is plotted as

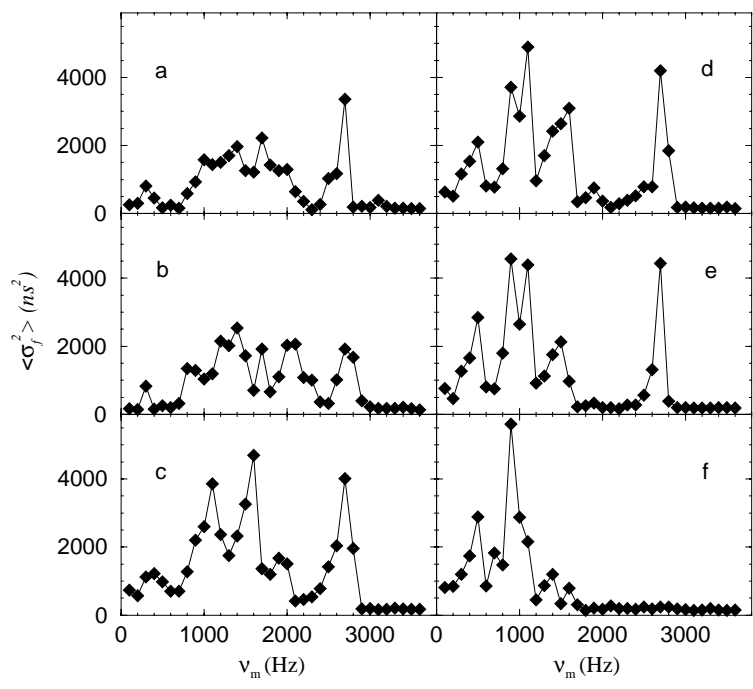

Figure 1: Averaged final rms beam size versus modulation frequency for various modulation amplitude (on the secondary rf cavity). This is done by averaging the rms beam size over the last $1 \mathrm{~ms}$ of the data taking $\left(<\sigma_{f}^{2}>\right)$. The alphabetic letters indicate the modulation amplitudes, $A_{2}$, where a: $60^{\circ}, \mathrm{b}: 100^{\circ}$, c: $125^{\circ}$, d: $150^{\circ}$, e: $175^{\circ}$, and f: $200^{\circ}$.

a function of the modulation frequency for the modulation amplitudes $60^{\circ}, 100^{\circ}, 125^{\circ}, 150^{\circ}, 175^{\circ}$, and $200^{\circ}$. Since our results indicate that the beam response appeared to be important at the modulation frequencies above and near the harmonics of the synchrotron frequency [4]. The data analysis is mostly focused on the modulation frequencies about integer factors of the synchrotron frequency. Fig. 2 shows that by properly adjusting the parameters, $f_{\mathrm{s}}, \Delta \phi_{0}$ and $\alpha$, the tracking results match quite well with the data.

The sensitivity of beam diffusion mechanism on the modulation frequency can be visualized by the FFT spectra of experimental data. Fig. 3 shows the FFT spectra of the rms beam widths of all data with a modulation amplitude of $150^{\circ}$. A direct response line is visible diagonally across the figure. Furthermore, the quadruple and the octuple modes arising from the dominant parametric resonances are particularly strong when the modulation frequencies are driven at $2 f_{\mathrm{s}}$ and $4 f_{\mathrm{s}}$. We note also that when the beam is driven into chaotic sea, the data show characteristic strong low frequency response.

\section{CONCLUSION}

Our experimental data show that the linear growth of mean square of beam width, $\left\langle\sigma^{2}\right\rangle$, with time arises from the diffusion process in a complete chaotic region in the phase space. If the phase space possess a layer of chaotic sea with invariant tori embedded inside, $\left\langle\sigma^{2}\right\rangle$ will show characteristics of anomalous diffusion. On the other hand, if stable islands still exist in the chaotic background as shown in $a$ and $f$ of Fig. 4, the evolution of $\left\langle\sigma^{2}\right\rangle$ will be strongly 


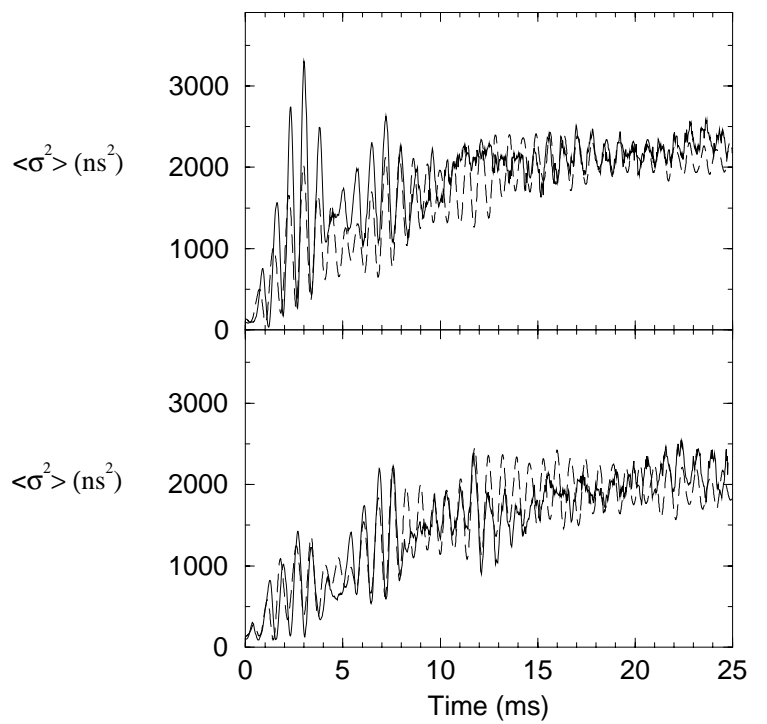

Figure 2: Comparison between data (solid curves) and simulation (dashed curves) for modulation on the secondary cavity near $2: 1$ resonance. The set parameters are $f_{m 2} \simeq 1200 \mathrm{~Hz}, A \simeq 100^{\circ}$ and $r \simeq 0.11$. The parameters used in tracking are $f_{\mathrm{s}}=719 \mathrm{~Hz}$. The only difference between the two figures is for the top one $\Delta \phi_{0}=215^{\circ}$ and for the bottom one $\Delta \phi_{0}=143^{\circ}$.

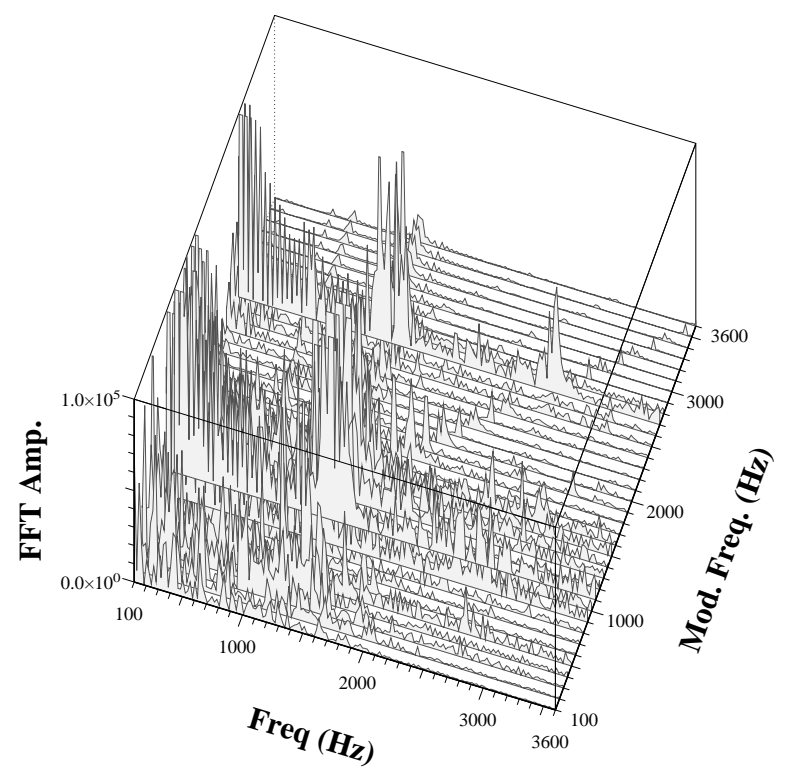

Figure 3: The FFT spectra of the experimental data of mean square beam widths for the modulation amplitude of $150^{\circ}$. We note that the low frequency response in the data arises from the chaotic sea, the quadruple and octuple modes arises from strong parametric resonances.

oscillatory. Our experiments, with numerical simulations, have systematically verified these conditions. The understanding of the signature of the beam phase space evolution can be used to diagnose sources of emittance dilution mechanisms in high brightness beams and space charge dominated beams.

We have experimentally measured the evolution of beam distribution as a function of rf parameters in a storage ring. These parameters are the ratio of rf voltages $r$, the modulation frequency $f_{\mathrm{m} 2}$, the modulation amplitude $A_{2}$, and the relative phase $\Delta \phi_{0}$. We have found that the evolution of the bunch beam can be divided into a fast process that is related to particle diffusion along the dominate parametric resonances, and a slow process that particles diffuse inside the chaotic sea.

We have also observed for the first time the coherent octuple excitation in beam. The corresponding phase space evolution for the octuple excitation has been clearly measured. Particles stream through the separatrix of the 4:1 parametric resonance, and then diffuse into the chaotic sea. The signature of the beam signal can easily be identified by comparing the data and tracking simulation shown in Fig.4, which also clearly indicates that the chaotic area strongly depends on $\Delta \phi_{0}$.

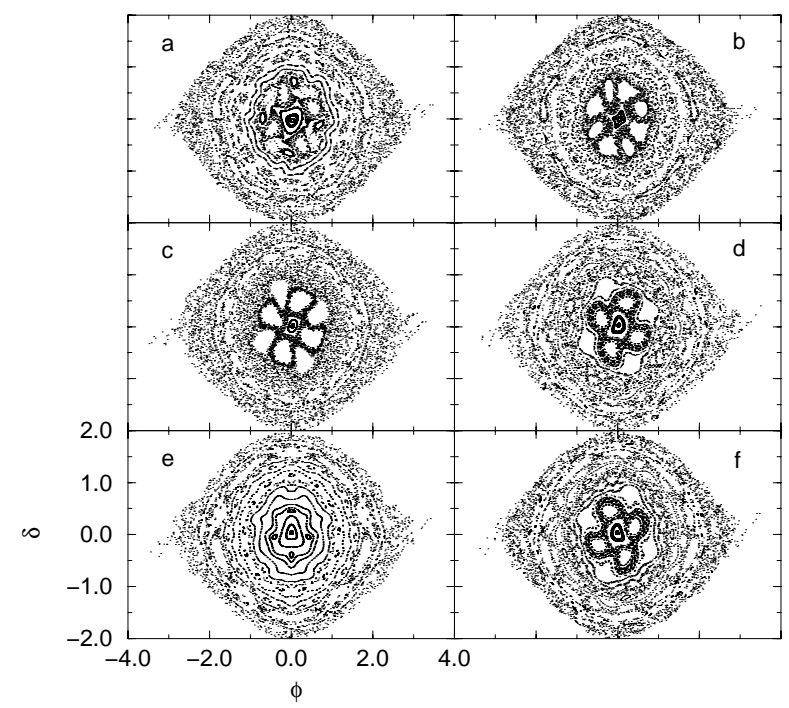

Figure 4: The Poincare surfaces of section for the modulation frequency of $2700 \mathrm{~Hz}$ with different relative phases, $\Delta \phi_{0}$, where a: $20^{\circ}$, b: $60^{\circ}$, c: $100^{\circ}$, d: $140^{\circ}$, e: $180^{\circ}$ and f: $220^{\circ}$. Note that the chaotic structure arises mainly from the overlapping resonances of 4:1 parametric resonances.

\section{REFERENCES}

[1] D. Jeon et al., Phys. Rev. Lett. 80, 2314 (1998).

[2] See e.g., C.L. Bohn, Phys. Rev. E 50, 1516 (1994); J. Struckmeier, Phys. Rev. E 54, 830 (1996) and references therein.

[3] M. Ellison et al., Phys. Rev. Lett. 70, 591 (1993); H. Huang et al., Phys. Rev. E48, 4678 (1993); D. Li et al., Phys. Rev. E48, R1638 (1993); D. Li et al., Nucl Inst. Methods A 364, 205 (1995).

[4] see e.g. S.Y. Lee, in Accelerator physics at the SSC, AIP Proceedings No. 326, edited by Y. Yan and M. Syphers, p.13 (AIP, N.Y. 1995). 